\title{
SCHWINGER TERMS, GERBES, AND OPERATOR RESIDUES
}

\author{
JOUKO MICKELSSON \\ Department for Theoretical Physics, Royal Institute of Technology, \\ S-10044 Stockholm, Sweden \\ E-mail: jouko@theophys.kth.se
}

1. Introduction. In these notes I want to describe recent developments concerning chiral anomalies and hamiltonian quantization, their relation to the theory of gerbes, extensions of generalized loop algebras using the residue calculus of pseudodifferential operators, and a new renormalization method in QFT.

In $1+1$ space-time dimensions the gauge current is a multiplication operator acting in the one-particle fermionic Hilbert space $H$. In particular, the time components of the current are infinitesimal generators for the group of gauge transformations. If the physical space is compactified as a circle, then this group is the loop group $L G$. A peculiar property of QFT in one space dimension is that most interesting operators (including the gauge current) can be second quantized using a standard normal ordering prescription. The mathematical reason for this is that the parts of the one-particle operators mixing positive and negative energy states are Hilbert-Schmidt.

The second quantized operators obey a slightly different algebra than their classical counterparts. In the process of quantization the Lie algebra $\mathbf{g l}_{1}$ of quantizable operators is promoted to a centrally extended Lie algebra $\widehat{\mathbf{g l}}_{1}$. The central extension is defined by a 2-cocycle, the Lundberg cocycle [Lu]. On the group level, we have a central extension of the group $G L_{1}$. This includes the loop group $L G$, leading to a central extension $\widehat{L G}$, the affine Kac-Moody group.

In higher than $1+1$ dimensions new problems arise. The simple normal ordering method is not sufficient to make the second quantized operators well-defined. Additional renormalization is needed. In the case of fermions in background gauge or gravitational fields the renormalization is achieved by a conjugation by unitary field dependent operators which reduce the size of the off-diagonal (with respect to the energy polarization) blocks of the observables. The size of the operators is best estimated by looking the

1991 Mathematics Subject Classification: Primary: 81T50, 17B65; Secondary: 81R10, 22E65. The paper is in final form and no version of it will be published elsewhere. 
asymptotic behavior, in momentum space, of the pseudodifferential symbols. In compact physical space of dimension $d$ a symbol is of Hilbert-Schmidt type iff it falls to zero faster than $|p|^{-d / 2}$ when $|p| \rightarrow \infty$.

After the initial renormalization in the one-particle space the operators can be quantized by normal ordering. The Lundberg cocycle leads to an extension of the original observable algebra. However, now the extension is not simply a central one because the renormalization is (external) field dependent and therefore the normal ordering subtraction depends also on the external fields. The resulting algebra is an extension of the original one by complex functions of the external fields. At first sight the cocycle is a very complicated expression in the fields. However, by writing the Lundberg cocycle in a cohomologically equivalent form, as a residue of an appropriate operator, we get a simple local expression. The reason is that the residue picks up only the terms which are of degree $-d$ in momentum space. The residue formula for the equivalent of Lundberg cocycle is closely related to the Radul cocycle. In fact, it can be viewed as a twisted version of the latter, the twist being defined by the sign of the energy, Theorem 5.13. Note that this is only a partial cocycle in the Lie algebra of PSDO's. The cocycle property is valid only for PSDO's $X$ satisfying the Hilbert-Schmidt condition on $[\epsilon, X]$.

The plan of these notes is the following. In Section 2 we shall go through some basic definitions in symbol calculus of PSDO's and the residue of a PSDO is defined and its fundamental properties are discussed. In Section 3 we discuss the Radul cocycle defining a central extension of the Lie algebra of PSDO's. The renormalization needed in higherdimensional setting is explained in Section 4 and is then applied in 5 for computing the structure of the quantized current algebra. In Section 6 we take a different, more geometric, point of view and study the structure of the bundle of fermionic Fock spaces parametrized by external fields with the help of the Atiyah-Patodi-Singer index theorem. Finally we explain the geometry of the bundle in terms of the theory of gerbes. This subsection is based on recent observations of A. Carey and M. Murray on the role of the Dixmier-Douady class of a bundle gerbe (this variant of the more general gerbe theory $[\mathrm{Br}]$ was introduced in $[\mathrm{Mu}]$ ) in quantization; actually, similar arguments were used previously in [Mi1] in the study of $3+1$-dimensional Schwinger terms but the role of the DD class was not understood at that time. The bridge between APS index theory and bundle gerbes was constructed in [CaMiMu].

2. Residue calculus of pseudodifferential operators. We shall consider differential and pseudodifferential operators defined in some domain in $\mathbb{R}^{n}$. A PSDO $A$ is defined by giving its symbol $a$. The symbol is a smooth matrix-valued function $a(x, p)$ of coordinates $x=\left(x_{1}, \ldots, x_{n}\right)$ and momenta $p=\left(p_{1}, \ldots, p_{n}\right)$. The action of $A$ onto a square integrable vector-valued function in $\mathbb{R}^{n}$ is given by

$$
(A \psi)(x)=\frac{1}{(2 \pi)^{n / 2}} \int a(x, p) e^{-i p \cdot x} \hat{\psi}(p) d p
$$

where

$$
\hat{\psi}(p)=\frac{1}{(2 \pi)^{n / 2}} \int e^{i p \cdot x} \psi(x) d x
$$


is the Fourier transform of $\psi$.

We shall consider only classical PSDO's; they have an asymptotic expansion, valid for $|p| \rightarrow \infty$,

$$
a(x, p) \sim \sum_{k=m, m-1, \ldots} a_{k}(x, p)
$$

where each $a_{k}$ is a homogeneous function of degree $k$ in the momenta, in the sense that $a_{k}(x, \lambda p)=\lambda^{k} a_{k}(x, p)$ for any real positive $\lambda$. The highest degree $m$ is the order of the PSDO $A$ and $a_{m}$ is its principal symbol. Any classical PSDO has a unique asymptotic expansion but two different PSDO's might have the same asymptotic expansion. The operator is unique up to addition by a infinitely smoothing operator $A$. The characteristic property of an infinitely smoothing symbol is that it approaches zero faster than inverse of any polynomial as $|p| \rightarrow \infty$. An example is the operator defined by the smooth symbol $\exp \left(-|p|^{2}\right)$.

Let $A, B$ be a pair of PSDO's with symbols $a, b$. The product $A B$ has a symbol $a * b$ which is given by the formula

$$
(a * b)(x, p)=\sum_{m} \frac{(-i)^{|m|}}{m !}\left(\partial_{p}^{m} a(x, p)\right)\left(\partial_{x}^{m} b(x, p)\right)
$$

where $m=\left(m_{1}, m_{2}, \ldots, m_{m}\right)$ are nonnegative integers, $|m|=m_{1}+\ldots m_{n}, m !=$ $m_{1} ! m_{2} ! \ldots m_{n} !, \partial_{p}^{m}=\left(\frac{\partial}{\partial p_{1}}\right)^{m_{1}} \ldots\left(\frac{\partial}{\partial p_{n}}\right)^{m_{n}}$, and similarly for $\partial_{x}^{m}$.

If $A$ is of order $k$ and $B$ is of order $\ell$ then $A B$ is of order $k+\ell$ and the principal symbol of $A B$ is the matrix product of the principal symbols of $A, B$. Note that the subleading term in the commutator $a_{k} * b_{l}-b_{l} * a_{k}$ is given by the Poisson bracket $-i\left\{a_{k}, b_{l}\right\}$.

A PSDO is a trace class operator if its order is strictly less than $-n$. The trace is then given by

$$
\operatorname{tr} A=\frac{1}{(2 \pi)^{n}} \int \operatorname{tr} a(x, p) d x d p
$$

the trace under the integral being the ordinary matrix trace. There is a useful alternative linear complex-valued functional on the algebra of PSDO's which also has the characteristic property that the 'trace' of a commutator is zero. This is the operator residue, due to V. Guillemin and M. Wodzicki [Gu], [Wo]. It is simply defined as

$$
\operatorname{Res}(A)=\frac{1}{(2 \pi)^{n}} \int_{|p|=1} \operatorname{tr} a_{-n}(x, p) d x d S(p)
$$

where $d S$ is the volume form on the sphere $|p|=1$. Alternatively,

$$
\operatorname{Res}(A)=\frac{1}{(2 \pi)^{n}} \int_{S} \operatorname{tr} a_{-n}(x, p) d x \theta(d \theta)^{n-1}
$$

where $\theta=\sum p_{i} d x_{i}$ is the symplectic 1-form and $S$ is any surface homotopic to the unit sphere in momentum space. This latter definition motivates the name operator residue. Let us restate the basic property

$$
\operatorname{Res}[A, B]=0
$$

for any pair of PSDO's. 
The operator residue is closely related to another form of trace: The Dixmier trace $\operatorname{tr}_{D}$. The latter is defined for operators with a spectrum such that

$$
\lim _{\Lambda \rightarrow \infty} \int_{|\lambda| \leq \Lambda}|\lambda| d \mu(\lambda)
$$

is (at most) logarithmically diverging, where $d \mu$ is the spectral measure. The Dixmier trace of a positive operator is then defined as the coefficient of $\log \Lambda$ in the above expression as $\Lambda \rightarrow \infty$. The case of a nonpositive operator needs more care but we shall not discuss that problem here because we shall need only (2.4).

Suppose now $A$ is a PSDO of order $-n$. By a simple computation one observes that each $a_{k}$ has a finite ordinary trace when $k<-n$. But the cut-off trace (momentum cut-off $|p| \leq \Lambda)$ of $a_{-n}$ behaves like $\log (\Lambda) \times$ const. for large $\Lambda$ where the constant is the integral of $a_{-n}(x, p)$ over the spherical directions in the momentum space (since $a_{-n}$ is a homogeneous function of degree $-n$ ), thus being equal to the residue of $A$. It follows that for PSDO's of degree $-n$ the Dixmier trace is the same as the residue. For trace class operators both the Dixmier trace and the residue vanish.

One can also write the operator residue as the complex residue of the zeta $\zeta_{A}$ function associated to the operator $A$. Recall that

$$
\zeta_{A}(s)=\int \lambda^{-s} d \mu(\lambda)
$$

provided that there is a spectral gap around zero. This definition is valid for positive PSDO's of nonzero order and for large real part of the complex variable $s$. By analytic continuation, the definition can be extended to the point $s=0$ and there one can define the zeta function regularized determinant $\log \left(\operatorname{det}_{\zeta} A\right)=\zeta_{A}^{\prime}(0)$. The definition can be even extended to nonpositive operator provided that there is a cone in the complex plane not intersecting with the spectrum of $A$, Friedlander [Fr], Kontsevich, Vishik [KoVi].

If $A$ is a PSDO of order $k$ then asymptotically $d \mu(\lambda) \sim|\lambda|^{(n-k) / k} d \lambda$ and therefore the zeta function has a pole at $s=n / k$. In particular, there is a pole at $s=-1$ (corresponding to naive trace of $A$ ) for $k=-n$. The residue at $s=-1$ is the residue of the operator $A$.

On a vector bundle over an arbitrary compact manifold a PSDO is an operator which in terms of local coordinates has a representation as (2.1), [Hö]. Even though the basic formulas of the symbol calculus have only local meaning, the residue still makes sense and has the cyclic property, [Wo].

3. The Radul cocycle. Let $\mathbf{g}$ be the Lie algebra of PSDO's on a compact manifold $M$. We define a central extension $\mathbf{g}^{\prime}=\mathbf{g} \oplus \mathbb{C}$ of $\mathbf{g}$ by the commutators

$$
[(A, \lambda),(B, \mu)]=([A, B], c(A, B))
$$

where $c$ is the Radul cocycle, [Ra] (the one-dimensional case was studied by Kravchenko and Khesin, $[\mathrm{KrKh}])$

$$
c(X, Y)=\operatorname{Res}[\log |p|, A] B .
$$

Here $|p|^{2}=\sum\left(p_{i}\right)^{2}$ is the symbol of the euclidean Laplace operator. Note that $\log |p|$ is not a classical PSDO but the commutator $[\log |p|, A]$ is. 
For any PSDO $A$ we have

$$
\operatorname{Res}[\log |p|, A]=0
$$

by integration in parts in configuration space. Therefore

$$
0=\operatorname{Res}[\log |p|, A B]=\operatorname{Res}[\log |p|, A] B+\operatorname{Res} A[\log |p|, B]
$$

from which it follows that

$$
\operatorname{Res}[\log |p|, A] B=-\operatorname{Res}[\log |p|, B] A
$$

and so $\omega(A, B)=-\omega(B, A)$. We have to prove the Jacobi identity for $\mathbf{g}^{\prime}$. This is equivalent with the 2-cocycle relation

$$
c(A,[B, C])+c(B,[A, C])+c(C,[A, B])
$$

To prove (3.2) we first use the antisymmetry of $\omega$,

$$
\begin{aligned}
c(A,[B, C]) & =\operatorname{Res}[\log |p|, A][B, C]=-\operatorname{Res}[\log |p|,[B, C]] A \\
& =-\operatorname{Res}[[\log |p|, B], C] A-\operatorname{Res}[B,[\log |p|, C]] A \\
& =-\operatorname{Res}[\log |p|, B][C, A]-\operatorname{Res}[\log |p|, C][A, B] .
\end{aligned}
$$

The right-hand side cancels exactly the last two terms in (3.2).

In one space dimension, when using the formula (2.4) for the residue, one usually modifies the definition of the cocycle:

$$
\omega(A, B)=\frac{1}{2} \operatorname{Res} \epsilon[\log |p|, A] B .
$$

Here $\epsilon=p /|p|$. In particular, if $A=A(x), B=B(x)$ are multiplication operators on the circle,

$$
\omega(A, B)=\frac{1}{2 \pi} \int \operatorname{tr} A^{\prime}(x) B(x)
$$

is the central term of an affine Kac-Moody algebra. We shall see that in applications to quantum field theory in higher space-time dimensions (3.3) is the correct formula to generalize and not the Radul formula (3.1).

4. Renormalization in higher dimensions. Consider a family of hamiltonians of the form $H(t)=D_{0}+A(t)$ acting in a one-particle Hilbert space $H$. We assume that $D_{0}$ is a time independent self-adjoint operator and $D_{0}+A$ is essentially self-adjoint in the same domain where $A$ is some bounded self-adjoint operator. We shall also assume that $A(t)$ has compact support in the time variable $t, A(t)=0$ for $|t|>T$. We study the time evolution equation

$$
i \partial_{t} U(t)=H(t) U(t), U(-T)=1 .
$$

Writing $V(t)=\exp \left(i t D_{0}\right) U(t) \exp \left(i T D_{0}\right)$ we obtain an equivalent equation

$$
i \partial_{t} V(t)=h(t) V(t) \text { with } V(-T)=1
$$

where $h(t)=e^{i t D_{0}} A(t) e^{-i t D_{0}}$. Since $h(t)$ is bounded and has finite support in $t$ this 
equation has a solution for all times given by the Dyson expansion

$$
V(t)=1+\sum_{n \geq 1}(-i)^{n} \int_{t>t_{n} \cdots>t_{1}>-T} \ldots \int h\left(t_{n}\right) \ldots h\left(t_{2}\right) h\left(t_{1}\right) d t_{n} \ldots d t_{2} d t_{1}
$$

Let $\epsilon=D_{0} /\left|D_{0}\right|$. We assume that zero is not an eigenvalue of $D_{0}$. The spectral decomposition $H=H_{+} \oplus H_{-}$corresponding to the splitting of the spectrum of $D_{0}$ to positive and negative parts fixes an irreducible representation of the canonical anticommutation relations $(\mathrm{CAR})$, uniquely defined up to equivalence, in a Fock space $\mathcal{F}$ with a vacuum $|0\rangle$ which is annihilated by the elements $a^{*}(v)$ and $a(u), v \in H_{-}, u \in H_{+}$, of the CAR algebra

$$
a^{*}(v) a\left(v^{\prime}\right)+a\left(v^{\prime}\right) a^{*}(v)=\left(v, v^{\prime}\right),
$$

and all the other anticommutators are equal to zero. Let $\left\{e_{n}\right\}_{n \in \mathbb{Z}}$ be an orthonormal basis of eigenvectors of $D_{0}$ such that the negative indices correspond to negative eigenvalues and nonnegative indices correspond to positive eigenvalues. Set $a_{n}=a\left(e_{n}\right)$ and $a_{n}^{*}=a^{*}\left(e_{n}\right)$. Fix the usual normal ordering for the products of creation and annihilation operators by $: a_{n}^{*} a_{m}:=-a_{m} a_{n}^{*}$ if $n=m<0$ and all the other products remain unchanged.

It is known that a bounded one-particle operator $A=\left(A_{n m}\right)$ can be canonically quantized as

$$
\hat{A}=\sum A_{n m}: a_{n}^{*} a_{m}:
$$

iff $[\epsilon, A]$ is Hilbert-Schmidt, for a survey on these matters see [Ar]. This quantization preserves the commutation relations of the Lie algebra of linear operators except for a complex-valued cocycle ('Schwinger term') $c_{L}$. The Lundberg's cocycle $c_{L}$ is given by, $[\mathrm{Lu}]$,

$$
c_{L}(X, Y)=\frac{1}{4} \operatorname{tr} \epsilon[\epsilon, X][\epsilon, Y]
$$

for a pair $X, Y$ of quantizable one-particle operators. The commutation relations of the corresponding quantum operators are

$$
[\hat{X}, \hat{Y}]=\widehat{[X, Y]}+c_{L}(X, Y) .
$$

In general, for the interaction term $A,[\epsilon, A]$ is not Hilbert-Schmidt, nor is $[\epsilon, U]$, and therefore the canonical quantum operator $\hat{U}$ does not exist.

We assume that the free hamiltonian $D_{0}$ is a self-adjoint PSDO of degree greater or equal to 1 . We also shall make the following additional assumptions:

(1) $A$ is bounded PSDO (thus of degree zero)

(2) $\operatorname{deg}\left[\left|D_{0}\right|, A\right]$ is less or equal to $\operatorname{deg} A$.

These properties hold for example when $D_{0}$ is a Dirac operator and $A=\gamma^{\mu} A_{\mu}$ is a gauge interaction term. Define an unitary operator

$$
T_{A}=\exp \left(\frac{1}{4}\left|D_{0}\right|^{-1}\left[D_{0}, A\right]\left|D_{0}\right|^{-1}\right) .
$$

Note that $T_{A}=1+K$, where the degree of $K$ is less or equal to $\operatorname{deg} A-1$. The operator $T_{A}$ takes a solution of the time evolution equation for the hamiltonian $H(t)$ to a solution of the equivalent hamiltonian $H^{\prime}(t)=T_{A} H(t) T_{A}^{-1}-i\left(\partial_{t} T_{A}\right) T_{A}^{-1}$. 
LEMma 4.9. With the above assumptions on the interaction $A$ the operator $\left[\epsilon, A^{\prime}\right]$ is a PSDO of degree $k-1$, where $A^{\prime}=H^{\prime}-D_{0}$ and the degree of $[\epsilon, A]$ is $k$.

Proof. Now

$$
A^{\prime}=T_{A} A T_{A}^{-1}-\left[D_{0}, T_{A}\right] T_{A}^{-1}-i\left(\partial_{t} T_{A}\right) T_{A}^{-1} .
$$

Modulo terms of degree $k-1$,

$$
\left[\epsilon, A^{\prime}\right]=[\epsilon, A]-\left[D_{0},\left[\epsilon, \frac{1}{4}\left|D_{0}\right|^{-1}\left[D_{0}, A\right]\left|D_{0}\right|^{-1}\right] .\right.
$$

On the other hand, the second term is equal to

$$
\begin{aligned}
\frac{1}{4}\left[\epsilon,\left|D_{0}\right|^{-1}\left[D_{0},\left[D_{0}, A\right]\right]\left|D_{0}\right|^{-1}\right] & =\frac{1}{4}\left[\epsilon,-2 \epsilon A \epsilon+\epsilon D_{0} A\left|D_{0}\right|^{-1}+\left|D_{0}\right|^{-1} A D_{0} \epsilon\right] \\
= & \frac{1}{4}[\epsilon,-2 \epsilon A \epsilon+2 A]+O(k-1)=[\epsilon, A]+O(k-1)
\end{aligned}
$$

and thus cancels the $O(k)$ part of the first term.

We can apply this method successively: Starting from some interaction $A_{0}=A$ such that $\operatorname{deg}[\epsilon, A]=k \leq 0$ we get a new interaction $A_{1}=A^{\prime}$ using the conjugation $T_{A}$. We can then insert $A_{1}$ as an argument to $T$ and obtain an unitary operator $T_{A_{1}}$. This defines again a new interaction $A_{2}$ such that $\operatorname{deg}\left[\epsilon, A_{2}\right] \leq k-2$. Continuing this way we obtain, after $p$ steps, an unitary operator $T_{A}^{(p)}=T_{A_{p-1}} \ldots T_{A_{0}}$ such that the time evolution for the operator $T_{A}^{(p)} U(t)$ is determined by an interaction $A_{p}$ such that $\left[\epsilon, A_{p}\right]$ is of degree $\leq k-p$. In particular, for $p>n$ the new interaction satisfies the Hilbert-Schmidt condition. Thus we have, [LaMi],

THEOREM 4.10. There is an unitary PSDO $T_{A}$, depending continuously on A, such that the time evolution $T_{A} U(t)$ satisfies the time evolution equation with the hamiltonian $H^{\prime}(t)=D_{0}+A^{\prime}$ such that $\left[\epsilon, A^{\prime}\right]$ is Hilbert-Schmidt. The operator $T_{A}$ is obtained as a product of the operators (4.8) obtained recursively from the initial A. Furthermore, at times $|t|>T$ (for which $A(t)=0$ ) the transformation $T_{A}$ is the identity operator.

This theorem was proven in [LaMi] for writing a simplified (compared with the original proofs of Palmer and Ruijsenaars [Pa], [Ru]) proof of the existence of the fermionic scattering matrix in external field problems. The renormalization which we introduced above has the important property that the time evolution operator for all intermediate times can be implemented in the free fermionic Fock space. This is in striking contrast with the more conventional approaches were only the asymptotic scattering operator is implementable. We are note going to pursue on the scattering problems any more here. Instead, we shall use our method in the next section for a renormalization of the current algebra and calculation of the Schwinger terms.

5. Schwinger terms. We shall assume that the physical space $M=\mathbb{R}^{n}$ and that the vector potentials, infinitesimal gauge transformations, and the symbols of all other PSDO's considered below fall off at $|x| \rightarrow \infty$ at least like $|x|^{-n / 2-\alpha}$ for some positive $\alpha$.

In this section we shall work in the hamiltonian formalism. At a fixed instant of time, $t=0$ say, the renormalization operator $T_{A}$ of the previous section becomes a time independent function of the potential $A(t=0)$. The hamiltonian is conjugated 
as $H^{\prime}=T_{A} H T_{A}^{-1}$. The currents (multiplication operator in the one-particle space) are also affected by the renormalization. Before the renormalization the action of a gauge transformation $g$ consists of a multiplication operator in the one-particle fermionic Hilbert space and the usual gauge action in the space of background vector potentials. After the renormalization in the one-particle space the new fermionic gauge action is given by the operator

$$
\Omega(g ; A)=T_{A} g\left(T_{A^{g}}\right)^{-1} .
$$

Here $A^{g}=g^{-1} A g+g^{-1} d g$ is the gauge transformed potential. By definition, $\Omega$ satisfies the one-cocycle equation

$$
\Omega\left(g g^{\prime} ; A\right)=\Omega(g ; A) \Omega\left(g^{\prime} ; A^{g}\right) .
$$

Differentiating $\Omega$ along one-parameter subgroups one obtains the operators $\theta(X ; A)=$ $T_{A} X T_{A}^{-1}-\left(\mathcal{L}_{X} T_{A}\right) T_{A}^{-1}$ for infinitesimal gauge transformations $X: M \rightarrow$ g. Here $\mathcal{L}_{X}$ is the Lie derivative in the direction of the infinitesimal gauge transformation $X$. They satisfy

$$
\theta([X, Y] ; A)=\mathcal{L}_{X} \theta(Y ; A)-\mathcal{L}_{Y} \theta(X ; A)+[\theta(X ; A), \theta(Y ; A)]
$$

Note that the modified Gauss law generators $G_{X}^{\prime}=\mathcal{L}_{X}+\theta(X ; A)$ satisfy the same commutation relations as the unrenormalized operators $G_{X}=\mathcal{L}_{X}+X$. This is because actually $G_{X}^{\prime}=T_{A} G_{X} T_{A}^{-1}$.

The operators $\theta(X ; A)$ satisfy

LemMa 5.4. $[\epsilon, \theta(X ; A)] \in L_{2}$.

Proof. Let $\epsilon(A)$ be the sign of the hamiltonian $D_{A}=D_{0}+A$; we use the abbreviation $A=A_{k} \gamma_{k}$. Here $\gamma_{k} \gamma_{l}+\gamma_{l} \gamma_{k}=2 g_{k l}$ is the usual $\gamma$-matrix algebra, $1 \leq k, l \leq n$. Since the commutator of the renormalized interaction $A^{\prime}$ with $\epsilon$ is Hilbert-Schmidt, the difference $\epsilon(A)-T_{A}^{-1} \epsilon T_{A}$ is also Hilbert-Schmidt.

But

$$
\begin{aligned}
{[\epsilon, \Omega(g ; A)] } & =\epsilon T_{A} g\left(T_{A^{g}}\right)^{-1}-T_{A} g\left(T_{A^{g}}\right)^{-1} \epsilon \\
& =T_{A}\left(\left(T_{A}{ }^{-1} \epsilon T_{A}\right) g-g\left(\left(T_{A^{g}}\right)^{-1} \epsilon T_{A}\right)\right) T_{A}{ }^{-1} \\
& \equiv T_{A}\left(\epsilon\left(A^{g}\right) g-g \epsilon(A)\right)\left(T_{A^{g}}\right)^{-1} \bmod L_{2}
\end{aligned}
$$

where $L_{2}$ is the ideal of Hilbert-Schmidt operators and we have used the gauge transformation rule $g^{-1} \epsilon(A) g=\epsilon\left(A^{g}\right)$. The statement for $\theta(X ; A)$ follows by taking the derivative with respect to a one-parameter subgroup.

From (5.4) it follows that the canonically quantized operators $\hat{\theta}(X ; A)$ exist. The second quantized Gauss law generators $\hat{G}_{X}^{\prime}=\hat{\theta}(X ; A)+\mathcal{L}_{X}$ act on wave functions $\psi(A)$ taking values in the free fermionic Fock space $\mathcal{F}$. The geometric meaning of the renormalization $T$ is this: The fermionic Fock spaces for each background field $A$ form a vector bundle over $\mathcal{A}$, the space of smooth external potentials. This bundle is trivial since the base is an affine space. Nevertheless, the representations of the CAR algebra in different fibers are inequivalent. The renormalization operator $T_{A}$ identifies the Fock space over $A$ with the free Fock space over $A=0$. 
Remark. We could take a more general point of view: Take $\mathcal{A}$ as the space of all perturbations of the free hamiltonian $D_{0}$ by bounded PSDO's satisfying. Assume that the symbol of $D_{0}$ is $p=p_{k} \gamma_{k}$ plus operators of order less or equal to zero, i.e., $D_{0}$ is a generalized Dirac operator. The gauge group $\mathcal{G}$ can be taken as the group of unitary PSDO's $g$ such that the principal symbol $g_{0}$ commutes with $p$; this includes in particular the group of ordinary gauge transformations. Lemma 5.4 remains valid in this general setting and the method below for the computation of the Schwinger terms can be applied.

The generators $\hat{G}_{X}^{\prime}$ satisfy the commutation relations

$$
\left[\hat{G}_{X}^{\prime}, \hat{G}_{Y}^{\prime}\right]=\hat{G}_{[X, Y]}^{\prime}+c(X, Y ; A)
$$

where

$$
c(X, Y ; A)=c_{L}(\theta(X ; A), \theta(Y ; A)) .
$$

From the definition the 2-cocycle property immediately follows:

$$
0=c(X,[Y, Z] ; A)+\mathcal{L}_{X} c(Y, Z ; A)+\text { cyclic permutations. }
$$

The trace of a PSDO on $\mathbb{R}^{n}$ is given as the integral of the total symbol,

$$
\operatorname{tr} A=\frac{1}{(2 \pi)^{n}} \int \operatorname{tr} a(x, p) d x d p
$$

where $a$ is the symbol of an operator $A$. Since the symbol is assumed to be smooth, the convergence of the integral depends only on the asymptotic properties of the symbol. Assuming that the $x$ integral exists, a sufficient condition for the (absolute) convergence of the trace is that the degree of the operator is strictly less than $-n$.

It is useful to extend the formula (5.8) to the case when the integral converges only conditionally in the following sense:

$$
\operatorname{tr}_{C} A=\lim _{\Lambda \rightarrow \infty} \int_{|p|<\Lambda} d p \int d x \operatorname{tr} a(x, p) .
$$

Suppose next that $A$ is a PSDO such that the pointwise trace of the homogeneous term $a_{-n}$ is a total derivative in momentum space, $\operatorname{tr} a_{-n}=\sum_{k=1}^{n} \frac{\partial f_{k}}{\partial p_{k}}$, each $f_{k}$ being homogeneous and of degree $-n+1$ in momenta. Then we can define a $\operatorname{trace} \operatorname{tr} A$ in the following way. For a finite value of the cut-off we have

$$
\operatorname{tr}_{\Lambda} A=\sum_{i \leq N} \Lambda^{i} \alpha_{i}(A) .
$$

In principle we should also have a logarithmically diverging term $\log (\Lambda) \cdot \alpha_{\log }(A)$ arising from the homogeneous symbol of degree $-n$. However, the assumption that this particular symbol is a total derivative leads instead to the finite expression

$$
\int_{|p|=\Lambda} \operatorname{tr} \frac{p_{k}}{|p|} f_{k}(p, x) d p d x
$$

which is independent of the value of the cut-off. It follows that we can define consistently

$$
\text { TR } A=\alpha_{0}(A) \text {. }
$$

For trace-class operators TR $A=\operatorname{tr} A$ is the standard trace. Furthermore, if $A$ is conditionally trace class then $\operatorname{tr}_{C} A=\mathrm{TR} A$. (Along these lines there is a theory of traces 
of PSDO's of nonintegral order in a more general setting on a compact manifold by Kontsevich and Vishik, [KoVi].)

LEMmA 5.12. Let $A, B$ be a pair of PSDO's on $\mathbb{R}^{n}$ (with the asymptotic $x$-space properties stated earlier $)$. Then $\operatorname{TR}[A, B]$ exists and is equal to $\operatorname{Res}[\log |p|, A] B$.

Pr o of. See [CFNW]. Note that TR $A$ does not exist for all operators $A$ and therefore the cocycle $\operatorname{TR}[A, B]$ is nontrivial.

ThEOREM 5.13. Let $\mathbf{b}$ be the Lie algebra of all PSDO's of degree zero such that $[\epsilon, X]$ is of degree less than $-n / 2$. Then the cocycle $c_{L}$ is well-defined on $\mathbf{b}$. The function $c(X, Y)=\frac{1}{2} \operatorname{Res} \epsilon[\log |p|, X] Y$ is also a 2-cocycle on $\mathbf{b}$, equivalent with $c_{L}$.

Proof. In this generality, the theorem was proven in [CFNW]; for the particular case of renormalized gauge currents it was proven earlier in [Mi2]. We give here a simpler proof. We can write

$$
\begin{aligned}
c_{L}(X, Y) & =\frac{1}{2} \operatorname{tr}_{C}[\epsilon, X] Y=\frac{1}{2} \operatorname{TR}[\epsilon, X] Y \\
& =\frac{1}{2} \operatorname{TR}([X \epsilon, Y]-\epsilon[X, Y])=\frac{1}{2} \operatorname{TR}[X \epsilon, Y]-\frac{1}{2} \operatorname{TR} \epsilon[X, Y] .
\end{aligned}
$$

The first term on the right is in the TR class because it is a commutator. Since we know that the sum is conditionally convergent, the second term is also in the TR class. We can define an extension $\mathrm{TR}^{\prime}$ of TR to the space of all PSDO's. This is of course not unique, but any extension will do. Define $\lambda(X)=\mathrm{TR}^{\prime} \epsilon X$. Obviously $\mathrm{TR} \epsilon[X, Y]$ is a coboundary of $\lambda$. Thus the Lundberg cocycle is cohomologous to the first term on the right in (5.14). Using (5.12) we obtain

$$
c_{L}(X, Y) \sim-\frac{1}{2} \operatorname{Res}[\log |p|, Y] X \epsilon=-\frac{1}{2} \operatorname{Res} \epsilon[\log |p|, Y] X=\frac{1}{2} \operatorname{Res} \epsilon[\log |p|, X] Y .
$$

Now we can apply this general formula to the case of renormalized current operators. For example, in three space dimensions and in the case of 2-component fermions we can choose $T_{A}$ such that it has the following asymptotic expansion:

$$
T_{A}=1+\frac{1}{4} \frac{[p, A]}{|p|^{2}}+O(-2) .
$$

Here $p=p_{k} \sigma_{k}$ and $A=A_{k} \sigma_{k}$ and the $\sigma_{k}$ 's are the hermitian Pauli matrices, $k=1,2,3$. We have dropped the lower order terms because they already are Hilbert-Schmidt. Of course, they must be included in order to preserve unitarity. For calculating Schwinger terms we do not need any terms which are of order less than -2 . This is because the residue in Theorem 5.13 depends only on the degree three term; on the other hand, the commutator with $\log |p|$ introduces one extra factor $1 /|p|$, so $X, Y$ need to be known only down to degree -2 .

The renormalized currents are given then as $(X, Y$ are now multiplication operators by Lie-algebra-valued functions) 


$$
\begin{aligned}
\theta(X ; A)=X+\frac{i}{4|p|^{2}}[p, d X] & -\frac{1}{4|p|^{2}}\left[\sigma_{k}, A\right] \partial_{k} X \\
& +\frac{[p, A]}{2|p|^{4}} p_{k} \partial_{k} X+\frac{[p, A]}{16|p|^{4}}[p, d X]+O(-3) .
\end{aligned}
$$

Here $d X=\sigma_{k} \partial_{k} X$. Inserting $\theta(X ; A)$ and $\theta(Y ; A)$ as the arguments in the cocycle, instead of $X, Y$, one obtains after a straightforward computation, [Mi2],

$$
c(X, Y ; A)=\frac{i}{24 \pi^{2}} \int \operatorname{tr} A \wedge(d X \wedge d Y-d Y \wedge d X) .
$$

This is the Faddeev-Mickelsson cocycle found in another context in [Mi3], [Mi4], [F], [F-Sh].

6. Gerbes, index theory, anomalies. In this section we want to describe a more geometric approach to the problem of construction of the family of Fock spaces parametrized by external vector potentials and the action of the gauge group. Actually, the method here is very general and applies as well to the case of an external metric field or any other interactions for that matter. In order to keep the discussion as simple as possible we shall restrict to the case of vector potentials.

The fermionic Fock spaces parametrized by Yang-Mills potentials form a vector bundle $\mathcal{F}$ over the space $\mathcal{A}$. In the case of chiral massless fermions there are subtleties in defining this bundle. The difficulty is related to the fact that the splitting of the one particle fermionic Hilbert space $H$ to positive and negative energies is not a continuous function of the external field. One can easily construct paths in the space of external fields such that at some point on the path a positive energy state dives into the negative energy space (or vice versa). These points are obviously discontinuities in the definition of the space of negative energy states and therefore the fermionic vacua do not form of smooth vector bundle over the space of external fields. This problem does not arise if we have massive fermions in the temporal gauge $A_{0}=0$. In that case there is a mass gap $[-m, m]$ in the spectrum of the Dirac hamiltonians and the polarization to positive and negative energy subspaces is indeed continuous.

If $\lambda$ is a real number not in the spectrum of the hamiltonian then one can define a bundle of fermionic Fock spaces $\mathcal{F}_{A, \lambda}^{\prime}$ over the set $U_{\lambda}$ of external fields $A, \lambda \notin \operatorname{Spec}\left(D_{A}\right)$. The vacuum in $\mathcal{F}_{A, \lambda}^{\prime}$ is defined by the polarization of the one-particle space to positive and negative spectrum of the operator $D_{A}-\lambda$. It turns out that the Fock spaces $\mathcal{F}_{A, \lambda}^{\prime}$ and $\mathcal{F}_{A, \lambda^{\prime}}^{\prime}$ are naturally isomorphic up to a phase. The phase is related to the arbitrariness in filling the Dirac sea between vacuum levels $\lambda, \lambda^{\prime}$. Such a filling is given corresponds (because of the anticommutation relations) to an exterior product $v_{1} \wedge v_{2} \wedge \ldots v_{m}$ of a complete orthonormal set of eigenvectors $D_{A} v_{i}=\lambda_{i} v_{i}$ with $\lambda<\lambda_{i}<\lambda^{\prime}$. A rotation of the eigenvector basis gives a multiplication of the exterior product by the determinant of the rotation. Thus there is a well-defined complex line $\operatorname{DET}_{\lambda \lambda^{\prime}}(A)$ for each $A \in U_{\lambda} \cap U_{\lambda^{\prime}}=$ $U_{\lambda \lambda^{\prime}}$ and

$$
\mathcal{F}_{A, \lambda^{\prime}}^{\prime}=\mathcal{F}_{A, \lambda}^{\prime} \otimes \operatorname{DET}_{\lambda \lambda^{\prime}}(A)
$$

over the intersection set. We set $\operatorname{DET}_{\lambda^{\prime} \lambda}=\operatorname{DET}_{\lambda \lambda^{\prime}}^{-1}$ for $\lambda<\lambda^{\prime}$. Note that from these 
definitions it follows immediately that the line $\operatorname{DET}_{\lambda \lambda^{\prime \prime}}$ can be naturally identified as $\mathrm{DET}_{\lambda \lambda^{\prime}} \otimes \mathrm{DET}_{\lambda^{\prime} \lambda^{\prime \prime}}$, i.e., the local line bundles $\mathrm{DET}_{\lambda \lambda^{\prime}}$ form a cocycle over the open cover $\left\{U_{\lambda}\right\}$ of $\mathcal{A}$. In order to compensate the dependence on $\lambda$ in the definition of the Fock spaces we search for a family of complex line bundles $\mathrm{DET}_{\lambda}$ over the open sets $U_{\lambda}$ such that

$$
\operatorname{DET}_{\lambda^{\prime}}=\operatorname{DET}_{\lambda^{\prime} \lambda} \otimes \operatorname{DET}_{\lambda}
$$

over $U_{\lambda \lambda^{\prime}}$. Obviously, the cocycle property of the of the line bundles $\operatorname{DET}_{\lambda \lambda^{\prime}}$ is a necessary condition for the existence of the family of bundles $\mathrm{DET}_{\lambda}$. It is not very hard to prove that this is also a sufficient condition, [Mi1]. This follows also form the general theory of bundle gerbes $[\mathrm{Mu}]$ since $\mathcal{A}$ is topologically trivial.

We define the tensor product

$$
\mathcal{F}_{A, \lambda}=\mathcal{F}_{A, \lambda}^{\prime} \otimes \operatorname{DET}_{A, \lambda} .
$$

Using (6.1) and (6.2) we observe that the right-hand side is independent from $\lambda$ and one has a well-defined bundle $\mathcal{F}$ of Fock spaces over all of $\mathcal{A}$.

Next one can ask what is the action of the gauge group in $\mathcal{F}$. The gauge action in $U_{\lambda}$ lifts naturally to $\mathcal{F}^{\prime}$. Thus the only problem is to construct a lift of the action on the base to the total space of $\mathrm{DET}_{\lambda}$. Note that the determinant bundle here is a bundle over external fields in odd dimension, and therefore one would expect that it is trivial (curvature equal to zero) on the basis of families index theorem. However, it turns out that the relevant determinant bundle actually comes from a determinant bundle in even dimensions. Instead of single vector potentials we must study paths in $\mathcal{A}$, thus the extra dimension. The relevant index theorem is then the APS theorem for even-dimensional manifolds with a boundary; physically, the boundary can be interpreted as the union of the space at the present time and in the infinite past, [CaMiMu].

We recall some facts about lifting a group action on the base space $X$ of a complex line bundle to the total space $E$. Let $\omega$ be the curvature 2-form of the line bundle. It is integral in the sense that $\int \omega$ over any cycle is $2 \pi \times$ an integer. Let $G$ be a group acting smoothly on $X$. Then there is an extension $\hat{G}$ which acts on $E$ and covers the $G$ action on $X$. The fiber of $\hat{G} \rightarrow G$ is equal to $\operatorname{Map}\left(X, S^{1}\right)$. As a vector space, the Lie algebra of the extension is $\mathbf{g} \oplus \operatorname{Map}(X, i \mathbb{R})$. The commutators are defined as

$$
[(a, \alpha),(b, \beta)]=\left([a, b], \omega(a, b)+\mathcal{L}_{a} \beta-\mathcal{L}_{b} \alpha\right)
$$

where $a, b \in \mathbf{g}$ and $\alpha, \beta: X \rightarrow i \mathbb{R}$. The vector fields generated by the $G$ action on $X$ are denoted by the same symbols as the Lie algebra elements $a, b$; thus $\omega(a, b)$ is the function on $X$ obtained by evaluating the 2 -form $\omega$ along the vector fields $a, b$. The Jacobi identity

$$
\omega([a, b], c)+\mathcal{L}_{a} \omega(b, c)+\text { cyclic permutations }=0
$$

for the Lie algebra extension $\hat{\mathbf{g}}$ follows from $d \omega=0$.

What we need is a formula for the curvature of the line bundles $\mathrm{DET}_{\lambda}$ along gauge directions. Not surprisingly, this is given by a reduction from a secondary characteristic class. Recall that in even space-time dimensions the Chern class of the determinant line bundle is obtained by starting from an appropriate characteristic class (the class appearing in the index formula of Dirac operators) in two higher dimensions and then 
integrating over the space-time manifold; this leaves a closed integral differential form of degree two on the parameter space of the Dirac operators, [AS]. In the odd-dimensional case here one starts from the APS index formula on a manifold with a boundary, [APS]. The formula contains two pieces on the right-hand side. The first is an integral of a local differential polynomial (the same as in the case without boundary) and the second is the so-called eta-invariant which contains nonlocal information about the spectrum of the boundary Dirac operator. The essential property of the eta-invariant is that it is gauge invariant. For that reason it does not give a contribution to the curvature of the determinant bundle along gauge orbits. Everything comes from the local differential polynomial; the non-gauge-invariant piece of the latter comes from the boundary and is equal to a secondary characteristic class. In simple situations this is just a Chern-Simons form.

Integrating the Chern-Simons form in $2 n+3$ dimensions over the $2 n+1$-dimensional physical space gives a 2 -form along gauge orbits.

For example, when $\operatorname{dim} M=1$, starting from the Chern-Simons form $\frac{1}{8 \pi^{2}} \operatorname{tr}(A d A+$ $\frac{2}{3} A^{3}$ ) we get

$$
\omega_{A}(X, Y)=\frac{1}{4 \pi} \int_{S^{1}} \operatorname{tr} A_{\phi}[X, Y]
$$

the curvature at the point $A$ in the directions of infinitesimal gauge transformations $X, Y$. (Note the normalization factor $2 \pi$ relating the Chern class to the curvature formula.) This is not quite the central term of an affine Kac-Moody algebra, but it is equivalent to it (in the cohomology with coefficients in $\operatorname{Map}(\mathcal{A}, \mathbb{C})$ ). In other words, there is a 1 -form $\theta$ along gauge orbits in $\mathcal{A}$ such that $d \theta=\omega-c$, where

$$
c(X, Y)=\frac{i}{2 \pi} \int \operatorname{tr} X \partial_{\phi} Y
$$

is the central term of the Kac-Moody algebra, considered as a closed constant coefficient 2 -form on the gauge orbits. There is a simple explicit expression for $\theta$,

$$
\theta_{A}(X)=\frac{i}{4 \pi} \int \operatorname{tr} A X
$$

When $\operatorname{dim} M=3$ the curvature (or equivalently, the Schwinger term) is obtained from the five-dimensional Chern-Simons form

$$
C S_{5}(A)=\frac{i}{24 \pi^{3}} \operatorname{tr}\left(A(d A)^{2}+\frac{3}{2} A^{3} d A+\frac{3}{5} A^{5}\right) .
$$

By the same procedure as in the one-dimensional case we obtain

$$
\omega_{A}(X, Y)=\frac{i}{4 \pi^{2}} \int \operatorname{tr}\left(\left(A d A+d A A+A^{3}\right)[X, Y]+X d A Y A-Y d A X A\right) .
$$

This differs from the FM cocycle

$$
\omega_{A}^{\prime}(X, Y)=\frac{i}{24 \pi^{2}} \int \operatorname{tr} A(d X d Y-d Y d X)
$$

by the coboundary of

$$
\frac{-i}{24 \pi^{2}} \int \operatorname{tr}\left(A d A+d A A+A^{3}\right) X
$$


The Dixmier-Douady class. Let $P \mathcal{F}$ be the bundle of projective Fock spaces $\mathcal{F}_{A} / \mathbb{C}^{\times}$ over $\mathcal{A}$. Because the action of $\mathcal{G}$ on $\mathcal{A}$ lifts to the total space $\mathcal{F}$ modulo $A$-dependent phases arising from the Schwinger terms the group $\mathcal{G}$ acts on $P \mathcal{F}$. The action of the subgroup $\mathcal{G}_{0}$ of based gauge transformations is free and therefore we can define the quotient bundle $P \mathcal{F} / \mathcal{G}_{0}$ over the manifold $\mathcal{A} / \mathcal{G}_{0}$. This projective bundle is nontrivial in the sense that there is no Hilbert bundle $\mathcal{H}$ over $\mathcal{A} / \mathcal{G}_{0}$ whose projectivization would be equal to $P \mathcal{F} / \mathcal{G}_{0}$, [Se]. The obstruction to constructing the Hilbert bundle is a certain element $\omega_{3} \in H^{3}\left(\mathcal{A} / \mathcal{G}_{0}, \mathbb{Z}\right)$, called the Dixmier-Douady class of the bundle, [Br]. The relation of the DD class to quantum field theory was recently clarified by Carey and Murray, [CaMu]; see also [CaMuWa]. I shall briefly describe the construction of $\omega_{3}$ below.

We need a locally finite partition of unity on $X=\mathcal{A} / \mathcal{G}_{0}$ subordinate to the covering by the open sets $V_{\lambda}=\pi\left(U_{\lambda}\right)$ where $\pi: \mathcal{A} \rightarrow \mathcal{A} / \mathcal{G}_{0}$ is the projection. On a locally compact manifold there exists always a partition of unity subordinate to a given open cover. However, in this case $X$ is not locally compact and we have no proof of the existence of the partition of unity. For that reason we assume that $X$ stands for any finite-dimensional submanifold of $\mathcal{A} / \mathcal{G}_{0}$ or any other submanifold such that there is a partition of unity $\left\{f_{\lambda}\right\}$ subordinate to the open sets $X \cap V_{\lambda}$. Let $\theta_{\lambda \lambda^{\prime}}$ be a representative for the Chern class of the bundle $\mathrm{DET}_{\lambda \lambda^{\prime}}$. Because of the cocycle property of the line bundles we can choose the 2 -forms $\theta_{\lambda \lambda^{\prime}}$ such that

$$
\theta_{\lambda \lambda^{\prime}}+\theta_{\lambda^{\prime} \lambda^{\prime \prime}}=\theta_{\lambda \lambda^{\prime \prime}}
$$

The forms $\theta_{\lambda \lambda^{\prime}}$ on $U_{\lambda \lambda^{\prime}}$ descend to forms on $V_{\lambda \lambda^{\prime}}$.

We define

$$
\theta_{\lambda}(x)=\sum_{\lambda^{\prime}} \theta_{\lambda \lambda^{\prime}}(x) f_{\lambda^{\prime}}(x)
$$

at points $x \in V_{\lambda}$. Now we have

$$
\theta_{\lambda}-\theta_{\lambda^{\prime}}=\sum_{\lambda^{\prime \prime}}\left(\theta_{\lambda \lambda^{\prime \prime}}-\theta_{\lambda^{\prime} \lambda^{\prime \prime}}\right) f_{\lambda^{\prime \prime}}=\sum_{\lambda^{\prime \prime}} \theta_{\lambda \lambda^{\prime}} f_{\lambda^{\prime \prime}}=\theta_{\lambda \lambda^{\prime}}
$$

by (6.8) and by $\sum f_{\lambda}(x)=1$, on the intersection $V_{\lambda \lambda^{\prime}}$. The forms $\theta_{\lambda}$ are not closed but on the intersection $V_{\lambda \lambda^{\prime}}$ we have $d \theta_{\lambda}=d \theta_{\lambda^{\prime}}$ since $\theta_{\lambda \lambda^{\prime}}$ is closed. Thus we may patch together the closed forms $d \theta_{\lambda}$ to a global closed form $\omega_{3}$ on $X$. This is the Dixmier-Douady class of the projective bundle $P \mathcal{F}$.

The above construction is an example of a bundle gerbe, introduced by Murray, [Mu] (which in turn is a specialization of the more general theory of gerbes, $[\mathrm{Br}]$ ). A bundle gerbe is defined as follows. Let $\pi: Y \rightarrow X$ be some fibration; in general this is not locally trivial, so $Y$ does not need to be a fiber bundle over $X$. Let

$$
Y^{[2]}=\left\{\left(y, y^{\prime}\right) \in Y \times Y \mid \pi(y)=\pi\left(y^{\prime}\right)\right\} .
$$

A bundle gerbe is a principal $\mathbb{C}^{\times}$bundle $P$ over $Y^{[2]}$ with a smooth associative composition map

$$
P_{(x, y)} \times P_{(y, z)} \rightarrow P_{(x, z)} .
$$

The bundle gerbe has also an identity (which is a section of $P$ over the diagonal $Y \subset Y^{[2]}$ ) and an inverse $P_{(x, y)} \rightarrow P_{(y, x)}, p \mapsto p^{-1}$. 
Example. Let $X=\mathcal{A}$ and $Y=\left\{(A, \lambda) \mid A \in \mathcal{A}, \lambda \notin \operatorname{Spec}\left(D_{A}\right)\right\} . \pi: Y \rightarrow X$ is the natural projection. In this case $Y^{[2]}=\left\{\left(A, \lambda, \lambda^{\prime}\right) \mid \lambda, \lambda^{\prime} \notin \operatorname{Spec}\left(D_{A}\right)\right\}$. The bundle $P$ over $Y^{[2]}$ is obtained as the collection of the line bundles $\operatorname{DET}_{\lambda \lambda^{\prime}}$ (with the zero section deleted) over the sets $\left\{\left(A, \lambda, \lambda^{\prime}\right) \mid A \in U_{\lambda \lambda^{\prime}}\right\} \subset Y^{[2]}$. Similarly, a curvature form $\theta$ for $P$ is obtained by patching together the local 2-forms $\theta_{\lambda \lambda^{\prime}}$. The fiber product is given by the natural identification of $\mathrm{DET}_{\lambda \lambda^{\prime}} \otimes \mathrm{DET}_{\lambda^{\prime} \lambda^{\prime \prime}}$ and $\mathrm{DET}_{\lambda \lambda^{\prime \prime}}$. Since the bundles $\mathrm{DET}_{\lambda \lambda^{\prime}}$ descend to $V_{\lambda \lambda^{\prime}}$ and the forms $\theta_{\lambda \lambda^{\prime}}$ are gauge invariant, the whole construction descends to the quotient by $\mathcal{G}_{0}$ producing a bundle gerbe over $\mathcal{A} / \mathcal{G}_{0}$.

In the above example the bundle gerbe over $\mathcal{A}$ is trivial (since $\mathcal{A}$ is flat), which means that

$$
P=\pi_{1}^{*}(L) \otimes \pi_{2}^{*}\left(L^{-1}\right)
$$

for some line bundle $L$ over $Y$. In our example $L$ is obtained by patching together the local bundles $\mathrm{DET}_{\lambda}$. However, the corresponding bundle gerbe over $\mathcal{A} / \mathcal{G}_{0}$ is nontrivial. The obstruction to trivializing $P / \mathcal{G}_{0}$ is given by the Dixmier-Douady class $\left[\omega_{3}\right]$.

In general, the DD class is constructed starting from the short exact sequence of de Rham complexes

$$
0 \rightarrow \Omega^{*}(X) \stackrel{\pi^{*}}{\rightarrow} \Omega^{*}(Y) \stackrel{\pi_{1}{ }^{*}-\pi_{2}^{*}}{\rightarrow} \Omega^{*}\left(Y^{[2]}\right) \cap \operatorname{Im}\left(\pi_{1}^{*}-\pi_{2}^{*}\right) \rightarrow 0 .
$$

This induces a long exact sequence in cohomology:

$$
\cdots \rightarrow H^{q}(X) \stackrel{\pi^{*}}{\rightarrow} H^{q}(Y) \stackrel{\pi_{1}{ }^{*}-\pi_{2}{ }^{*}}{\rightarrow} H_{\pi}^{q}\left(Y^{[2]}\right) \stackrel{\Delta}{\rightarrow} H^{q+1}(X) \rightarrow \ldots
$$

where $H_{\pi}^{q}$ denotes the image of $\pi_{1}{ }^{*}-\pi_{2}{ }^{*}$. The form $\omega_{3}$ which we constructed above is actually equal to $\Delta(\theta)$. However, the same reservation applies to the use of the exact sequence as we had before in the construction of $\omega_{3}$ : The construction of the map $\Delta$ uses a locally finite open cover, so strictly speaking it is valid only in the case of locally compact manifolds.

Finally, I want to mention an important topic closely related to the problems discussed in these notes but which were omitted here because of lack of time and space. The structure of the current algebra and anomalies in gauge theory can be described in a more general setting of Fredholm modules, incorporating ideas from noncommutative geometry, [Co]. This was initiated in [MiRa] (see also the monograph [Mi4]) and generalized even further in recent articles, see [La1], [La2] and references therein.

Acknowledgements. I wish to thank A. Carey, D. Burghelea, E. Langmann, and S. N. M. Ruijsenaars for discussions at various stages in preparing these lecture notes. I also want to thank G. Marmo and P. Michor for inviting me to the Erwin Schrödinger Institute, where the main part of this work was written.

\section{References}

[Ar] H. Araki, Bogoliubov automorphisms and Fock representations of canonical anticommutation relations, in: Operator algebras and mathematical physics, Contemp. Math. 62, Amer. Math. Soc., Providence, 1987, 23-141. 
[APS] M. F. Atiyah, V. K. Patodi, and I. M. Singer, Spectral asymmetry and Riemannian geometry, I-III, Math. Proc. Cambridge Philos. Soc. 77 (1975), 43-69; 78 (1975), 405-432; 79 (1976), 71-79.

[AS] M. F. Atiyah, I. M. Singer, Dirac operators coupled to vector potentials, Proc. Nat. Acad. Sci. U.S.A. 81 (1984), 2597-2600.

[Br] J.-L. Brylinski, Loop Spaces, Characteristic Classes, and Geometric Quantization, Birkhäuser, Boston-Basel-Berlin, 1993.

[CaMiMu] A. L. Carey, J. Mickelsson, M. Murray, Index theory, gerbes, and quantization, Comm. Math. Phys. (to appear); hep-th/9511151.

[CaMu] A. L. Carey and M. K. Murray, Mathematical remarks on the cohomology of gauge groups and anomalies, Internat. J. Modern Phys. A (to appear); hep-th/9408141.

[CaMu1] A. L. Carey and M. K. Murray, Faddeev's anomaly and bundle gerbes, Lett. Math. Phys. 37 (1996), 29-36.

[CaMuWa] A. L. Carey, M. K. Murray and B. Wang, Higher bundle gerbes, descent equations and 3-Cocycles, preprint 1995.

[CFNW] M. Cederwall, G. Ferretti, B. Nilsson, and A. Westerberg, Schwinger terms and cohomology of pseudodifferential operators, Comm. Math. Phys. 175 (1996), 203-220; hep-th/9410016.

[Co] A. Connes, Noncommutative Geometry, Academic Press, San Diego, 1994.

[F] L. Faddeev, Operator anomaly for the Gauss law, Phys. Lett. B 145 (1984), 81.

[F-Sh] L. Faddeev and S. Shatashvili, Algebraic and Hamiltonian methods in the theory of nonabelian anomalies, Theoret. Math. Phys. 60 (1984), 770.

[Fr] L. Friedlander, Ph.D. thesis, Dept. of Math., M.I.T., 1989.

[Gu] V. Guillemin, A new proof of Weyl's formula on the asymptotic distribution of eigenvalues, Adv. Math. 55 (1985), 131.

[Hö] L. Hörmander, The Analysis of Partial Differential Operators, III, Springer, Berlin, 1985.

[KoVi] M. Kontsevich, S. Vishik, Determinants of elliptic pseudo-differential operators, hep-th/9404046.

[KrKh] O. S. Kravchenko and B. A. Khesin, A nontrivial central extension of the Lie algebra of pseudodifferential symbols on the circle, Functional Anal. Appl. 25 (1991), 83.

[La1] E. Langmann, Noncommutative integration calculus, J. Math. Phys. 36 (1995), $3822-3835$.

[La2] E. Langmann, Descent equations of Yang-Mills anomalies in noncommutative geometry, hep-th/9508003.

[LaMi] E. Langmann and J. Mickelsson, Scattering matrix in external fields, J. Math. Phys. 37 (1996), 3933-3953.

[Lu] L.-E. Lundberg, Quasi-free second quantization, Comm. Math. Phys. 50 (1976), 103.

[Mi1] J. Mickelsson, On the hamiltonian approach to commutator anomalies in $3+1$ dimensions, Phys. Lett. 241 (1990), 70-76.

[Mi2] J. Mickelsson, Wodzicki residue and anomalies of current algebras, in: Integrable models and strings, ed. A. Alekseev et al., Lecture Notes in Phys. 436, Springer, Berlin, 1994, 123-135. 
[Mi3] J. Mickelsson, Chiral anomalies in even and odd dimensions, Comm. Math. Phys. 97 (1985), 361-370.

[Mi4] J. Mickelsson, Current Algebras and Groups, Plenum Press, London and New York, 1989.

[MiRa] J. Mickelsson and S. Rajeev, Current algebras in $d+1$ dimensions and determinant bundles over infinite-dimensional Grassmannians, Comm. Math. Phys. 116 (1988), 365-400.

[Mu] M. K. Murray, Bundle gerbes, J. London Math. Soc. (2) (to appear); dg-ga/9407015.

[Pa] J. Palmer, Scattering automorphisms of the Dirac field, J. Math. Anal. Appl. 64 (1978), 189.

[Ra] A. O. Radul, Lie algebras of differential operators, their central extensions, and W-algebras, Functional Anal. Appl. 25 (1991), 33.

[Ru] S. N. M. Ruijsenaars, Gauge dependence and implementability of the S-operator for spin-0 and spin- $\frac{1}{2}$ particles in time-dependent external fields, J. Funct. Anal. 33 (1979), 47.

[Se] G. Segal, unpublished preprint, Dept. of Math., Oxford University, 1985.

[Wo] M. Wodzicki, Noncommutative residue. I: Fundamentals, in: K-theory, arithmetic and geometry, ed. Yu. I. Manin, Lecture Notes in Math. 1289, Springer, Berlin, 1984, 320-399. 